\title{
REVISÃO SISTEMÁTICA DE LITERATURA E METASSÍNTESE QUALITATIVA: CONSIDERAÇÕES SOBRE SUA APLICAÇÃO NA PESQUISA EM ENFERMAGEM ${ }^{1}$ \\ SYSTEMATIC REVIEW OF LITERATURE AND QUALITATIVE METASYNTHESIS: CONSIDERATIONS ABOUT THEIR APPLICATION IN NURSING RESEARCH REVISIÓN SISTEMÁTICA DE LITERATURA Y METASÍNTESIS CUALITATIVA: CONSIDERACIONES SOBRE SU APLICACIÓN EN LA INVESTIGACIÓN EN ENFERMERÍA
}

\author{
Ana Lúcia Mendes Lopes², Lislaine Aparecida Fracolli
}

\begin{abstract}
${ }^{1}$ Este trabalho é parte da dissertação de mestrado apresentada ao Programa de Pós-graduação de Enfermagem em Saúde Coletiva da Escola de Enfermagem da Universidade de São Paulo (USP), em 2008.

${ }^{2}$ Mestre em Enfermagem em Saúde Coletiva. Enfermeira do Hospital Universitário da USP. São Paulo, Brasil.

${ }^{3}$ Doutora em enfermagem. Docente do Departamento de Enfermagem em Saúde Coletiva da Escola de Enfermagem da USP. Orientadora da dissertação. São Paulo, Brasil.
\end{abstract}

PALAVRAS-CHAVE: Pesquisa metodológica em enfermagem. Literatura de revisão como assunto. Pesquisa qualitativa.

KEYWORDS: Nursing methodology research. Review literature as topic. Qualitative research.

PALABRAS CLAVE: Investigación metodológica en enfermería. Literatura de revisión como assunto. Investigación cualitativa.
RESUMO: Trata-se de uma reflexão sobre a condução de metassíntese qualitativa, que aborda características da revisão bibliográfica sistemática, como uma tendência emergente da necessidade de reunir dados para a tomada de decisão em saúde, comparando as diversas modalidades de revisão, metanálise e metassíntese. Aborda também a pesquisa qualitativa, suas definições, variedade de disciplinas, métodos e peculiaridades, destacando a vasta produção em pesquisa qualitativa no âmbito da enfermagem, e a necessidade de dar visibilidade e impacto aos seus resultados, visando a sua aplicação na prática. Apresenta a definição de metassíntese qualitativa e suas características, discutindo aspectos relacionados à complexidade da metodologia e à sua condução prática. Conclui que a metassíntese qualitativa pode ser uma ferramenta a ser pesquisada no âmbito da Enfermagem, aperfeiçoando a metodologia e validando-a, através da inclusão da grande produção de pesquisa qualitativa com aspectos importantes do ofício de cuidar e ser cuidado.

ABSTRACT: This study is a reflection on the conduction of qualitative meta-synthesis. It addresses the characteristics of systematic bibliographic review as an emerging trend in gathering data for decisionmaking in health care, comparing diverse review methods, meta-analysis, and meta-synthesis. It discusses qualitative research through its definitions, various disciplines, methods and particularities, highlighting the vast production of qualitative research in nursing and the need create visibility and the impact of results as practical applications. This study presents a definition for qualitative metasynthesis and its characteristics, discussing the complexity of the methodology and practical aspects of the process. We conclude that qualitative meta-synthesis can be a tool to be researched in nursing, improving and validating the methodology through the inclusion of the large production of qualitative research in nursing, with key points of the profession of providing and receiving care.

RESUMEN: Se trata de una reflexión sobre la conducción de metasíntesis cualitativa, en la que se abordan características de la revisión bibliográfica sistemática, como una tendencia emergente de la necesidad de reunir datos para la toma de decisiones en salud, comparando diversas modalidades de revisión, metaanálisis y metasíntesis. También se aborda la investigación cualitativa, sus definiciones, variedad de disciplinas, métodos y peculiaridades, destacando la producción en investigación cualitativa en la enfermería, y la necesidad de dar visibilidad e impacto a sus resultados, teniendo como objetivo su aplicación práctica. Se presenta la definición de metasíntesis cualitativa y sus características, discutiendo aspectos relacionados con la complejidad de la metodología y su conducción práctica. Se concluye que la metasíntesis puede ser una herramienta a ser investigada en el ámbito de la enfermería, perfeccionando la metodología y validándola a través de la inclusión de la vasta producción de la investigación cualitativa en enfermería con importantes aspectos del oficio de cuidar y ser cuidado. 


\section{REVISÃO BIBLIOGRÁFICA SISTEMÁ- TICA}

O uso da revisão bibliográfica sistemática como fonte de evidência para organizar o crescente número de produtos, intervenções e informações científicas vem aumentando rapidamente, substituindo a pesquisa primária nas tomadas de decisão na área da saúde. ${ }^{1}$

A revisão bibliográfica sistemática é definida "como uma síntese de estudos primários que contém objetivos, materiais e métodos claramente explicitados e que foi conduzida de acordo com uma metodologia clara e reprodutível" .:672

As revisões sistemáticas reúnem grande quantidade de resultados de pesquisas clínicas, discutindo diferenças entre estudos primários que tratam do mesmo objeto. Os estudos primários são, neste caso, os sujeitos da pesquisa. ${ }^{3}$

Como o nome sugere, tais revisões são sistemáticas na abordagem e usam métodos explícitos e rigorosos para identificar textos, fazer apreciação crítica e sintetizar estudos relevantes. Exigem planejamento prévio e documentação através de protocolo. ${ }^{4}$

Esta metodologia fundamenta-se no Movimento de Pesquisa Baseada em Evidências, que emergiu do aumento da produção científica mundial, do crescente número de intervenções, tecnologias, medicamentos e terapias na área de saúde, e da necessidade de validar os resultados obtidos a partir de vários estudos sobre determinada questão, a fim de subsidiar a tomada de decisão, seja clínica, gerencial, política, seja epidemiológica. ${ }^{1,4}$

Ressalta-se que esta abordagem difere, amplamente, das revisões bibliográficas narrativas convencionais, uma vez que demandam uma seqüência de etapas cuja metodologia é claramente explicitada, com técnicas padronizadas e passíveis de reprodução. ${ }^{1-5}$

Entre as principais características da revisão sistemática estão: fontes de busca abrangentes, seleção dos estudos primários sob critérios aplicados uniformemente e avaliação criteriosa da amostra.

Quando a integração de estudos primários é sintetizada, mas não combinada estatisticamente, a revisão pode ser chamada de revisão sistemática qualitativa. ${ }^{5} \mathrm{O}$ tratamento estatístico dado aos resultados obtidos a partir de revisões sistemáticas é conhecido como meta-análise e vem sendo amplamente utilizado por pesquisadores, em todo o mundo.
O incremento desta metodologia deve-se, principalmente, aos estudos desenvolvidos pela Fundação Cochrane, iniciativa internacional com origem no Reino Unido, que desde 1992 prepara, mantém e dissemina revisões sistemáticas de intervenções de saúde, tornando-se a maior referência da pesquisa baseada em evidências. ${ }^{3}$

Esta modalidade de pesquisa tem, como foco, confirmar a efetividade de uma intervenção, freqüentemente através de estudos experimentais, como os estudos randomizados controlados. Embora tenham grande importância para a saúde, em geral, e para a enfermagem, em particular, tais estudos tendem a não incluir, em suas amostras bibliográficas, métodos interpretativos, observacionais ou descritivos, característica de grande parte da produção científica em enfermagem. ${ }^{1}$ No entanto, a experiência clínica, baseada na reflexão e no julgamento pessoal, é também necessária para a tradução de evidências científicas na intervenção e tratamento dos pacientes. ${ }^{1,6}$

Tomando como exemplo a evidência científica de que determinado medicamento ou terapia é efetivo, a sua indicação torna-se, certamente, mais segura, uma vez que a possibilidade de sucesso na sua utilização é bastante alta. Porém, tal evidência pode não dar pistas de como o paciente (grupo ou população) receberá esta terapia - se há recursos financeiros, se é adequado à sua rotina (familiar ou dos profissionais de saúde), à sua cultura, se o uso é confortável ou de difícil adesão. Tais temas, quando associados a mudanças de hábito e de estilo de vida, ou à inserção social, exigem modos diferentes de abordagem. ${ }^{1,6}$

Trata-se de perguntas diferentes que requerem diferentes tipos de pesquisa. ${ }^{1,6}$ É preciso utilizar ferramentas que possam trazer à luz as contradições, transformando-as em conhecimento. Neste sentido, os métodos qualitativos podem relacionar a evidência científica à prática clínica, no contexto do dia-a-dia. As pesquisas qualitativas estão, cada vez mais, contribuindo para o Movimento de Práticas Baseadas em Evidências. ${ }^{7}$

\section{PESQUISA QUALITATIVA}

As diversas profissões da saúde, especialmente a enfermagem, transitam predominantemente nas relações interpessoais e de assistência à saúde e doença. Por isso encontram coerência na interação humana e no aprofundamento das experiências individuais possibilitadas pela pesquisa qualitativa. ${ }^{8}$ 
Originada da pesquisa antropológica e sociológica, a pesquisa qualitativa é a denominação para uma "complexa e interconectada família de termos, conceitos e hipóteses", 9:4 provenientes de várias disciplinas e áreas de conhecimento. É definida genericamente como: “[...] atividade determinada que coloca o observador no mundo. Consiste num cenário de práticas interpretativas que torna o mundo visível. Estas práticas transformam o mundo. Elas tornam o mundo uma série de representações, incluindo notas de campo, entrevistas, diálogos, fotografias, gravações e memórias pessoais. Nesta perspectiva, pesquisa qualitativa envolve uma abordagem interpretativa e naturalística do mundo". $9: 3$

Ao se particularizar a pesquisa qualitativa para o campo clínico, destaca-se o cenário das vivências em saúde, definindo o "método clínicoqualitativo" em pesquisa "aquele que busca interpretar os significados - de natureza psicológica e complementarmente sociocultural - trazidos por indivíduos (pacientes ou outras pessoas preocupadas ou que se ocupam com problemas da saúde, tais como familiares, profissionais de saúde e sujeitos da comunidade), acerca dos múltiplos fenômenos pertinentes ao campo dos problemas da saúde-doença". 10:510

A pesquisa qualitativa também é entendida como capaz de "incorporar a questão do significado e da intencionalidade como inerentes aos atos, às relações, e às estruturas sociais, sendo estas últimas tomadas tanto no seu advento quanto na sua transformação, como construções humanas significativas". 11:10

A pesquisa qualitativa busca explorar "como as pessoas dão sentido ao mundo que as cerca, quem são elas, e como elas apresentam isto e respondem aos outros". ${ }^{7}$ A complexidade da pesquisa qualitativa advém do fato de não haver uma estratégia própria e única para a sua condução metodológica e interpretativa. Não há um paradigma ou teoria única que a caracterize. São várias as perspectivas teóricas que reivindicam a pesquisa qualitativa: construtivismo, estudos culturais, feminismo, marxismo e modelos étnicos de estudos. ${ }^{11}$

Seus métodos e práticas também variam: pesquisadores qualitativos usam a semiótica, narrativa, conteúdo, análise de fonemas, ou mesmo de estatísticas, gráficos, tabelas. São utilizadas técnicas e abordagens metodológicas provenientes da etnometodologia, fenomenologia, hermenêutica, psicanálise e observação participativa, entre outras. ${ }^{11}$

No campo da Enfermagem, destaca-se o amplo alcance dos variados temas abordados nas pesquisas qualitativas, que incluem construções pessoais e culturais sobre doença, prevenção e risco, bem como experiências de vida sob determinadas intervenções ou doenças crônicas. ${ }^{4}$

As possibilidades da pesquisa qualitativa são reconhecidas na investigação de atitudes, crenças e preferências de profissionais e pacientes. Seu valor está na habilidade de buscar, sistematicamente, respostas a perguntas que não são facilmente respondidas pela metodologia experimental. ${ }^{6}$

A produção de pesquisa qualitativa em saúde aumentou significativamente. ${ }^{6,12}$ Muitas revistas científicas divulgam estudos qualitativos, inclusive, revistas internacionais especializadas. ${ }^{10}$ As bases de dados eletrônicas indicam muitos estudos (teses, artigos, livros) conduzidos sob o desenho da pesquisa qualitativa.

Embora ainda seja necessário usar diferentes estratégias de busca para alcançar todos os estudos disponíveis, conforme as características de cada base de dados, ao acessar a interface da Biblioteca Virtual em Saúde em 21 de fevereiro de 2008 com o descritor "pesquisa qualitativa", observou-se haver 5.047 estudos na MEDLINE; 1.148, na base LILACS; 420, na base SciELO; além de 596 e 322 nas bases especializadas, como BDENF e ADOLEC, o que evidencia o crescente interesse pela área. A base CINAHL* identifica 32.975 estudos.

No entanto, esta produção não parece refletir-se com a mesma intensidade e impacto nas aplicações práticas. ${ }^{4-12}$ Uma das estratégias para que isto aconteça pode ser situar tal conhecimento em um contexto interpretativo mais amplo, apresentando-o de forma mais prática e aplicável junto ao "mundo real dos planejadores das políticas e das práticas". ${ }^{12: 365}$

Assim, através da necessidade de apreciação e de tornar visíveis estudos qualitativos clinicamente relevantes, surge o interesse na condução de revisões bibliográficas sistemáticas com a integração de estudos qualitativos.

\footnotetext{
* Biblioteca Virtual em Saúde, [página na Internet] disponível em: http://www.bireme.br/php/index.php. Base CINAHL [página na Internet] disponivel em: http://web.ebscohost.com/ehost/results?vid=7Ehid=105Esid= 46a40ee3-5277-4e6f-8db1-03035da96b1d\%40sessionmgr106.
} 


\section{METASSÍNTESE QUALITATIVA}

O tratamento de resultados qualitativos obtidos em uma revisão sistemática pode ser apresentado na forma narrativa, quantitativa de estatística de achados qualitativos (metassumarização), ou através da interpretação dos resultados (metassíntese). ${ }^{13}$

A metassumarização qualitativa é uma reunião de resultados qualitativos apresentados de modo quantitativamente orientados, na forma de tópicos ou sumários temáticos de surveys ou dados, como a soma de partes dos resultados sobre determinado tema. ${ }^{14}$

Eles indicam o conteúdo manifesto nos resultados e refletem a lógica quantitativa: discernir a freqüência de cada resultado, apontando os de maior prevalência, de modo a validá-los. Envolve a extração, além da abstração dos achados e do cálculo da amplitude da prevalência manifesta.

Metassíntese qualitativa é uma integração interpretativa de resultados qualitativos que são, em si mesmos, a síntese interpretativa de dados, incluindo fenomenologia, etnografia, teoria fundamentada nos dados, bem como outras descrições, coerentes e integradas, ou explanações de determinados fenômenos, eventos, ou de casos que são as marcas características da pesquisa qualitativa. ${ }^{14}$ Tais integrações vão além da soma das partes, uma vez que oferecem uma nova interpretação dos resultados. Essas interpretações não podem ser encontradas em nenhum relatório de investigação, mas são inferências derivadas de se tomar todos os artigos em uma amostra, como um todo.

As metassínteses oferecem descrição coerente ou explanação sobre determinado evento ou experiência. Tais integrações interpretativas exigem que o pesquisador capte as sínteses que constituem os resultados de relatórios de pesquisas individuais unidos para alinhavar uma ou mais metassínteses. Sua validade não está em uma replicação lógica, mas sim numa lógica integradora, cujas conclusões são acomodadas no artesanato exposto no produto final.

Os métodos da metassíntese incluem constante comparação, análise taxonômica, tradução recíproca de conceitos in vivo, bem como a utilização de conceitos importados para enquadrar dados. ${ }^{14}$

A metassíntese qualitativa originou-se da sociologia e pode ser definida como "uma modalidade de estudo qualitativo que utiliza os dados dos achados de outros estudos qualitativos relativos ao mesmo tema, ou a temas correlacionados" ${ }^{8: 312}$
A primeira abordagem metassintética publicada por enfermeiras foi um artigo (denominado meta-análise qualitativa) que interpreta os resultados de sete estudos, visando desenvolver um modelo para orientar enfermeiros na avaliação de mulheres para o autocuidado. ${ }^{8}$

Outras denominações para metassíntese (meta-synthesis ou metasynthesis) observadas na literatura são: meta-estudo (meta-study), meta-etnografia (meta-ethnography), meta-análise qualitativa (qualitative meta-analisys) e aggregate analysis.-15

Em 1998, a necessidade de desenvolver as melhores abordagens para a revisão de estudos qualitativos levou a Fundação Cochrane a estabelecer o Qualitative Research Methods Working Group - do qual vários estudiosos de pesquisa qualitativa em saúde participam como membros ou consultores - com a finalidade de desenvolver e dar suporte metodológico à inclusão de dados qualitativos em revisões sistemáticas, disponibilizando guias temáticos, aulas e instrumentos para a condução de revisões bibliográficas de pesquisas qualitativas, dentro do contexto da Fundação Cochrane. ${ }^{1,16}$

As enfermeiras Margareth Sandelowski e Julie Barroso, professoras da University of North Carolina at Chapel Hill School of Nursing e da Duke University School of Nursing, respectivamente, vêm desenvolvendo e divulgando conhecimentos sobre técnicas analíticas e interpretativas na integração de resultados de pesquisas qualitativas em revisões bibliográficas sistemáticas, construídos do conhecimento resultante de pesquisa ( $Q u a-$ litative Metasynthesis Project) ${ }^{17}$ sobre mulheres com sorologia positiva para o vírus HIV (no qual desenvolveram estas técnicas), e em estudos sobre mulheres e casais que receberam diagnóstico positivo durante o período pré-natal (quando refinaram as técnicas).

Para dar início ao projeto, elas utilizaram os procedimentos tradicionalmente associados a sínteses através de revisão bibliográfica sistemática, como critérios de inclusão, acesso e busca de literatura relevante e os procedimentos disponíveis sobre como avaliar estudos qualitativos. Os conhecimentos desenvolvidos foram divulgados em várias publicações. ${ }^{4,12-15,18}$ Além disso, essas profissionais criaram um manual para conduzir os estudos integrativos de pesquisa qualitativa, com guias interativos on-line referentes a capítulos do manual. ${ }^{19}$

Também coordenaram um projeto do National Institute of Nursing Research denominado Analytic Techniques for Qualitative Metasynthesis, com uma 
biblioteca virtual, periodicamente atualizada, que contém todos os estudos do projeto original. ${ }^{14}$

Estas pesquisadoras consideram metassíntese qualitativa "um tipo distinto de estudo em que os resultados de estudos qualitativos completos de um determinado campo são combinados". 4:154 O termo refere-se tanto ao produto interpretativo quanto aos processos analíticos nos quais tais estudos são agregados, integrados, resumidos ou mesmo reunidos.

A metassíntese qualitativa tem o propósito de criar traduções interpretativas ampliadas de todos os estudos examinados em determinado domínio, de modo que seu resultado seja fiel à tradução interpretativa de cada estudo em particular. ${ }^{4}$

Trata-se da interpretação do pesquisador (synthesist) sobre as interpretações dos dados primários, feitas por seus autores originais dos estudos que compõem a amostra da metassíntese que, por sua vez, é composta por estudos qualitativos distintos, selecionados com base em sua relevância para uma questão de pesquisa específica formulada por este pesquisador. ${ }^{8}$

Estes estudos não incluem sínteses narrativas ou revisões de estudos quantitativos, nem os resultados de análises secundárias de dados combinados a partir de estudos qualitativos separados. ${ }^{12}$ Diferentemente da metanálise, que é agregativa e reduz os dados a uma única unidade, a metassíntese acarreta em comparação, tradução e análise dos dados originais que resultam em novas interpretações, englobando e destilando os significados nos estudos constituintes da amostra. ${ }^{8}$

Embora "[...] possa ser considerada análoga à meta-análise, como 'um interesse compartilhado de sintetizar estudos empíricos' bem como no mesmo desejo de usar uma abordagem sistemática, inclusiva e comunicável na integração entre pesquisas, na metassíntese qualitativa não se calculam médias ou se reúnem resultados dentro de um mesmo intervalo de medidas, e sim se criam amplas traduções interpretativas de todos os estudos examinados". 4:154

Procurar reunir todo o conteúdo disponível sobre determinada temática pode ajudar a compreender os fenômenos e ampliar o conhecimento, favorecendo a sua aplicação na adoção de políticas e práticas, e nas tomadas de decisões no âmbito da saúde.

Sob esta perspectiva, a condução de estudos através da abordagem proposta pela revisão bibliográfica sistemática, seguida do tratamento dos resultados por meio da metassíntese qualitativa, pode possibilitar maior visibilidade e impacto às inúmeras pesquisas qualitativas conduzidas no âmbito da enfermagem.

\section{Considerações sobre a complexidade meto- dológica da metassíntese qualitativa}

Vários pesquisadores chamam a atenção para os aspectos caracterizadores e importantes da pesquisa qualitativa que correm o risco de ser perdidos, na tentativa de agrupar ou resumir seus resultados, devido a grande variedade de disciplinas, políticas, teorias, filosofias, princípios éticos e metodologias envolvidos em tais pesquisas. ${ }^{1,8,12}$

Por isso, não se pode, simplesmente, resumir a integridade de cada projeto individual, que reflete a subjetividade e a complexidade da experiência humana exclusiva sobre saúde e doença, nem as características emancipatórias e pedagógicas inerentes a cada estudo qualitativo.

Pesquisas qualitativas contêm informações sobre as "sutilezas e complexidades das respostas à doença e seu tratamento, que são essenciais à construção de efetivas intervenções sensíveis ao desenvolvimento e à cultura" ${ }^{18: 782}$ Contemplam, ainda, conhecimentos referentes à profunda compreensão dos profissionais de saúde sobre a experiência vivida, a partir da perspectiva de seus clientes, dentro de contextos sociais, históricos e culturais específicos. ${ }^{8}$

Tal argumento, associado ao fato de a pesquisa qualitativa conter diferentes modos de coleta, análise e interpretação de dados, como já comentado, poderia contra-indicar a integração de pesquisas qualitativas. Porém, justifica-a, uma vez que os resultados obtidos podem influenciar o conhecimento, proporcionando tomadas de decisões que considerem tais aspectos, essenciais à vida humana. ${ }^{1,7,18}$

Portanto, "esforços no sentido de se sintetizar estudos qualitativos são vistos como essenciais para atingir proposições analíticas mais elevadas e também ampliar a generalização da pesquisa qualitativa" ${ }^{12: 367}$ Não se considera, aqui, a generalização como baseada em amostragem e significância estatística, mas como uma generalização sobre particularidades, que cria amplas interpretações de todos os estudos examinados, de modo a se permanecer fiel a cada um, em particular. ${ }^{4}$

Sob este aspecto, é possível considerar a metassíntese qualitativa como a "interpretação multivocal de um fenômeno", cujas vozes de par- 
ticipantes diferentes podem estar em um mesmo estudo. ${ }^{8: 315}$ Entretanto, tais participantes não têm a mesma história, crenças ou visão de mundo, tampouco as metodologias ou os pesquisadores individuais compartilham a mesma filosofia e propostas. O que relaciona as pesquisas, os participantes, as metodologias e os pesquisadores dos estudos primários, entre si, é o fato de participarem do mesmo fenômeno estudado, através da análise e da interpretação do revisor.

Portanto, o pesquisador da metassíntese precisa ser cuidadoso, dada a complexidade metodológica dos estudos primários e o contexto dos pesquisadores originais de cada estudo. ${ }^{8}$

\section{Tipos de metassíntese qualitativa e questões para a sua condução}

Encontram-se descritas na literatura três estratégias para a síntese de resultados de estudos qualitativos. A primeira envolve a integração de resultados de múltiplos caminhos desenvolvidos em um programa de pesquisa por um mesmo investigador(es). ${ }^{12}$

A segunda consiste na síntese dos resultados de pesquisas de diferentes investigadores, integradas por técnicas como análise comparativa qualitativa, tradução recíproca de metáforas chave (categorizando a informação obtida dos dados individuais em "variáveis-chave"), análise de conteúdo, entre outras. ${ }^{12}$

A terceira envolve o uso de métodos quantitativos para reunir resultados qualitativos de diferentes estudos, de modo a transformá-los em dados passíveis de análise estatística (metassumarização). ${ }^{12}$

Um dos dilemas fundamentais na condução da metassíntese qualitativa é a decisão sobre quais estudos tratam, realmente, do mesmo fenômeno, evento ou experiência. ${ }^{12} \mathrm{~A}$ utilização de técnicas adequadas na busca de informação, verificando as propostas do estudo primário, suas questões e os tipos de resultados produzidos, pode trazer à tona as diferentes facetas estudadas de um mesmo recorte, auxiliar a compreender diferentes linguagens e permitir selecionar os estudos que tratem do mesmo aspecto do fenômeno desejado para a síntese.

É importante considerar que cada aspecto pode permitir a condução de uma nova metassíntese (que podem ser combinadas no final), daí a importância de se decidir quantos aspectos serão tratados na metassíntese, uma vez que quanto mais pesquisas originais houver na amostra, menos profundas serão, geralmente, a análise e interpretação dos resultados. ${ }^{18}$

Além de decidir sobre a similaridade temática dos estudos incluídos na amostra, o pesquisador precisa determinar a sua comparabilidade metodológica, reconhecendo as semelhanças e diferenças entre os estudos selecionados.

Também se preconiza a comparação de características que incluam o tipo de literatura utilizada na revisão, o recorte do estudo, incluindo as técnicas usadas na seleção da amostra, coleta de dados e análise. Além de permitir ao pesquisador da metassíntese compreender cada relatório de pesquisa em sua individualidade (antes da integração ou comparação dos mesmos), essa observação possibilita reconhecer cada pesquisa e seu pesquisador original na sua singularidade, ou seja, seu background ou experiências. ${ }^{12}$

No que concerne à qualidade dos estudos primários, de modo geral, não se deve excluir estudos por razões de qualidade ${ }^{12}$ uma vez que as concepções do que é considerado "bom", nos critérios de qualidade, sofrem variações. Embora possa haver pequenos enganos superficiais, resultados valiosos podem ser desprezados ao se excluir algum estudo primário em nome do preciosismo metodológico. Ressalta-se, porém, que os estudos selecionados ainda "precisam obedecer a padrões de qualidade, como vivacidade descritiva, precisão analítica, relevância heurística e congruência metodológica". 12:368 As diferenças encontradas na qualidade metodológica dos estudos devem ser apontadas pelo sintetizador ao descrever as características de cada estudo.

Um dos critérios de avaliação de qualidade que pode ser utilizado é o formulário padronizado Critical Appraisal Skills Programme (CASP) (C) Milton Keynes Primary Care Trust, 2002) para pesquisas qualitativas. ${ }^{22}$

Um dos mais complexos problemas enfrentados pelo revisor é o desenvolvimento e a comunicação das técnicas utilizadas para comparar os resultados de cada estudo. No entanto, considerando-se que a razão de ser da metassíntese qualitativa está, exatamente, na reunião dos resultados, ressalta-se que "[...] não importa qual método é utilizado, o objetivo da metassíntese qualitativa é levar em conta toda importante similaridade e diferença na linguagem, nos conceitos, nas imagens e noutras idéias em torno de determinada experiência... ampliando as possibilidades interpretativas dos resultados e construindo narrativas ampliadas ou teorias gerais". ${ }^{12: 369}$ 


\section{CONSIDERAÇÕES SOBRE A APLICABI- LIDADE DA METODOLOGIA}

O desenho e a operacionalização da metassíntese, propriamente dita, necessitam de disponibilidade, sendo mais adequados a projetos em grupo - no qual um mesmo banco de dados pode oferecer material para pesquisa de diversos aspectos de um mesmo tema, além de reunir experts para a validação dos resultados.

As bases de dados, com suas diferentes formas de interação com o usuário, podem dificultar e retardar o processo. Há pesquisas qualificadas como qualitativas que são quantitativas, e repetição de títulos. A utilização de programas organizadores de citações pode ser uma alternativa facilitadora. Na análise, os programas de transcrição de textos ou de discursos também podem auxiliar no tratamento dos dados.

Pesquisadores mais experientes selecionam os artigos mais rapidamente. $\mathrm{O}$ sistema de busca sistemática de triagem e seleção por etapas - título, abstract, e artigo na íntegra, auxilia o pesquisador a ter uma aproximação gradual do denso conteúdo, diminuindo o risco de este enganar-se, eliminando ou incluindo os artigos errados. ${ }^{18}$

Uma das principais dificuldades enfrentadas pelo revisor é o repertório teórico, necessário ao reconhecimento de pesquisa qualitativa, e a identificação das diversas metodologias e modos de análise e interpretação que estão sob esta nomenclatura. A agilidade na análise e na tomada de decisão ocorre na medida em que se pratica a metodologia, sendo esta, em si, a melhor forma de aprender. A validação da seleção e da avaliação de qualidade por pares e experts é recomendada e aumenta a confiabilidade e transparência do processo de busca, da seleção de dados, análise e interpretação. ${ }^{12}$

Partindo do conhecimento gerado e validado pelo movimento de pesquisas baseadas em evidências, os pesquisadores qualitativos vêm desenvolvendo uma abordagem metodológica mais aproximada das características multidisciplinares e multiteóricas da metassíntese qualitativa.

A metassíntese qualitativa tem o potencial de ampliar o alcance dos resultados advindos da percepção, sentimentos, visão, vivência e experiência dos sujeitos. Pode contribuir para destacar os achados predominantes na produção científica selecionada, auxiliando decisões políticas, gerenciais e assistenciais em saúde, de modo geral, e na enfermagem, mais especificamente.
Como metodologia situada no âmbito da saúde e da enfermagem, tem relevância para dar visibilidade à produção científica qualitativa, característica de tantas pesquisas conduzidas por enfermeiros. A metassíntese qualitativa pode ser uma ferramenta de ampliação do conhecimento relativo a determinado tema.

Por esta razão, pode ser interessante desenvolver estudos sob este paradigma, considerandoo como possibilidade de aprendizado e de construção crítica, respeitando a necessidade de preparo do pesquisador para desenvolver a análise.

Desenhar um estudo sob a perspectiva da metassíntese qualitativa é uma empreitada desafiadora e atraente, tanto pela necessidade de aprender o método, quanto pela própria natureza do recorte da pesquisa qualitativa, no qual o estudo é desenhado enquanto é conduzido. ${ }^{15}$

As técnicas estão em desenvolvimento ${ }^{7-20} \mathrm{e}$ há uma tendência em denominá-la "integração de pesquisa qualitativa", em vez de metassíntese, pois esta denominação destaca a interpretação única do autor da síntese sobre os achados de outros autores. ${ }^{21}$ Apontam para a possibilidade de se organizar análises e interpretações sobre resultados qualitativos, de modo a proporcionar visibilidade e maior impacto que possam fornecer elementos para a tomada de decisão em saúde.

Sob esta perspectiva, é uma ferramenta a ser pesquisada no âmbito da enfermagem, visando aperfeiçoar a metodologia e validá-la, também pela vasta produção de pesquisa qualitativa existente em enfermagem, com aspectos importantes do ofício de cuidar e ser cuidado.

\section{REFERÊNCIAS}

1. Evans D, Pearsons A. Systematic reviews: gatekeepers of nursing knowledge. J Clin Nurs. 2001 Sep; 10(4):593-9.

2. Greenhalgh T. Papers that summarise other papers (systematic review and meta-analyses). BMJ. 1997 Sep; 13(315):672-5.

3. Castro AA, Saconato H, Guidugli F, Clark OAC. Curso de revisão sistemática e metanálise [texto na Internet]. São Paulo (SP): LED-DIS/UNIFESP; 2002. [acesso em 2008 Fev. 21] Disponível em: http:// www.virtual.epm.br/cursos/metanalise.

4. Barroso J, Gollop CJ, Sandelowski M, Meynell, Pearce PF, Collins LJ. The Challenges of Searching for and Retrieving Qualitative Studies. West J Nurs Res. 2003 Mar; 25(2):153-78.

5. Cook DJ, Mulrow CD, Raynes RB. Systematic reviews: synthesis of best evidence for clinical 
decisions. Ann Intern Med. 1997 Mar [acesso em 2008 Fev. 21]; 126(5):376-80. Disponível em: http:// www.annals.org/cgi/content/full/126/5/376.

6. Green J, Britten N. Qualitative research and evidence based medicine. BMJ. 1998 Apr 18; 316(7139):1230-2.

7. Flemming K, Briggs M. Eletronic Searching to locate qualitative research: evaluation of three strategies. J Adv Nurs. 2007 Jan; 57(1):95-100. Review.

8. Zimmer L. Qualitative meta-synthesis: a question of dialoging with texts. J Adv Nurs. 2006 Feb; 53(3):311-18.

9. Denzin NK, Lincoln YS. Handbook of qualitative research. Thousand Oaks (CA): Sage Publications; 2000. p.1-28.

10. Turato ER. Métodos qualitativos e quantitativos na área de saúde: definições, diferenças e seus objetos de pesquisa. Rev Saúde Pública. 2005 Jun; 39(3):507-14.

11. Minayo MCS. O desafio do conhecimento: pesquisa qualitativa em saúde. $8^{a}$ ed. São Paulo (SP): Hucitec; 2004. p.9-18.

12. Sandelowski M, Docherty S, Emden C. Focus on qualitative methods. Qualitative Metasynthesis: issues and techniques. Res Nurs Health. 1997 Aug; 20(4):365-71.

13. Sandelowski M, Barroso J. Classifying the findings in qualitative studies. Qual Health Res. 2003 Sep; 13(7):905-23.

14. Sandelowski M, Barroso J. Sandbar Digital Library Project. Qualitative metasummary method [página na Internet]. Chapel Hill (USA): University of North Carolina at Chapel Hill School of Nursing; 2004 [acesso em 2008 Fev. 21]. Disponível em: http:/ / sonweb.unc. edu/sandbar/index.cfm?fuseaction=about\#.
15. Sandelowski M, Barroso J. Focus on research methods: toward a metasynthesis of qualitative findings on motherwood in HIV-positive women. Res Nurs Health. 2003 Apr; 26(2):153-70. Review.

16. Cochrane Qualitatives Research Methods Group. Proposal to establish a Cochrane Qualitative Methods Group July 4th 2002 [texto na Internet]. Lancaster (UK): Cochrane, 2002 [acesso em $2008 \mathrm{Fev}$. 21]. Disponível em: http://www.joannabriggs.edu. au/cqrmg/documents/cochrane_proposal.pdf.

17. University of North Carolina at Chapel Hill School of Nursing. Qualitative Metasynthesis Project [página na Internet]. Chapel Hill (USA): UNC; 2008 [acesso 2008 Fev. 21]. Disponível em: http://www.unc. edu/ msandelo/qmp.

18. Sandelowski M, Barroso J. Writing the proposal for a qualitative research methodology project. Qual Health Res. 2003 Jul; 13(6):781-820.

19. D'Auria J, Barroso J, Sandelowski M. Synthesizing Qualitative Research [página na Internet]. Chapel Hill (UK): University of North Carolina at Chapel Hill School of Nursing; 2005 [acesso em 2007 Maio 7]. Disponível em: http:/ / www.unc.edu/ msandelo/ handbook/index.html.

20. Jones, ML. Aplication of systematic review methods to qualitative research: pratical issues. J Adv Nurs. 2004 Nov; 48(3):271-8.

21. Thorne S, Jensen L, Kearney MH, Noblit G e Sandelowski M. Qualitative metasynthesis: reflections on methodological orientation and ideological agenda. Qual Health Res. 2004 Dec; 14(10):1342-65.

22. Milton Keynes Primary Care Trust. Critical Appraisal Skills Programme (CASP). Making sense of evidence. London (UK): Oxford; 2002. 